\title{
Diagnostic Utility of Cytomegalovirus Nucleic Acid Testing during Antigenemia-Guided Cytomegalovirus Monitoring after Hematopoietic Stem Cell Transplantation or Liver Transplantation
}

\author{
Yoshitomo Morinaga, ${ }^{1}$ Yasushi Sawayama, ${ }^{2}$ Masaaki Hidaka, ${ }^{3}$ Sayaka Mori, ${ }^{1}$ \\ Jun Taguchi, ${ }^{2}$ Mitsuhisa Takatsuki, ${ }^{3}$ Susumu Eguchi, ${ }^{3}$ Yasushi Miyazaki ${ }^{2}$ and \\ Katsunori Yanagihara ${ }^{1}$ \\ ${ }^{1}$ Department of Laboratory Medicine, Nagasaki University Graduate School of Biomedical Sciences, Nagasaki, \\ Nagasaki, Japan \\ ${ }^{2}$ Department of Hematology, Atomic Bomb Disease and Hibakusha Medicine Unit, Atomic Bomb Disease \\ Institute, Nagasaki University, Nagasaki, Nagasaki, Japan \\ ${ }^{3}$ Department of Surgery, Nagasaki University Graduate School of Biomedical Sciences, Nagasaki, Nagasaki, \\ Japan
}

Cytomegalovirus (CMV) is an opportunistic pathogen, and careful monitoring of CMV is important for immunocompromised patients. Antigenemia-based CMV monitoring is a standard test used for managing CMV infection in transplant recipients; however, in Japan, there are no reports of CMV monitoring using the standardized test. The utility of a standardized CMV nucleic acid test (NAT) was evaluated during antigenemia-based CMV monitoring after hematopoietic stem cell transplantation (HSCT) or liver transplantation. Blood collection for CMV monitoring was performed under the physician's instructions depending on the condition of the patient, and CMV NAT and antigenemia was evaluated. For HSCT recipients, blood collection only for NAT was additionally performed during the pre-engraftment phase. The results of the NAT were blinded to those evaluating the results. A total of 34 patients were enrolled (11 HSCT recipients and 23 liver transplant recipients). NAT detected the first CMV episode no later than antigenemia in $2(18.2 \%)$ HSCT recipients and $3(13.0 \%)$ liver transplant recipients, earlier than antigenemia in $3(27.3 \%)$ HSCT recipients and $7(30.4 \%)$ liver transplant recipients, and later than antigenemia in $1(9.1 \%)$ HSCT recipient and 1 (4.3\%) liver transplant recipient. In 5 HSCT recipients, NAT was positive during the pre-engraftment phase. Among the 468 blood samples which were evaluated by both NAT and antigenemia, $124(26.7 \%)$ were positive in NAT and $51(10.9 \%)$ were positive in antigenemia. The standardized CMV NAT is useful for accurately diagnosing CMV infection and determining appropriate therapeutic interventions for HSCT recipients and liver transplant recipients.

Keywords: automation; immunocompromised; monitoring; nucleic acid test; pre-engraftment phase Tohoku J. Exp. Med., 2019 March, 247 (3), 179-187. (C) 2019 Tohoku University Medical Press

\section{Introduction}

Cytomegalovirus (CMV) is one of the most common opportunistic pathogens. Although CMV is generally acquired during childhood and infects a variety of cells including epithelial cells, macrophages, and lymphocytes, these infections are typically asymptomatic in healthy people. However, for immunocompromised patients such as transplant recipients, careful management of CMV infections is important because of the risk of acquiring hepatitis, pneumonia, colitis and retinitis due to CMV.
CMV antigen detection in peripheral blood leukocytes has been used as a standard method for determining the risk of CMV infections in Japan. A CMV antigenemia assay was conducted by observing infected leukocytes immunostained with monoclonal antibodies against $\mathrm{CMV}$ antigens such as $\mathrm{C} 10 / \mathrm{C} 11$ and pp65. However, CMV antigenemia has several limitations in terms of the handling process. For instance, handling in the pre-analysis stage can strongly affect the quality of the results because the test requires fresh leukocytes. It is difficult to standardize the testing process because this method is influenced by reading vari-

Received January 8, 2019; revised and accepted March 6, 2019. Published online March 19, 2019; doi: 10.1620/tjem.247.179.

Correspondence: Yoshitomo Morinaga, M.D., Ph.D., Department of Laboratory Medicine, Nagasaki University Graduate School of Biomedical Sciences, 1-7-1 Sakamoto, Nagasaki, Nagasaki 852-8501, Japan.

e-mail: y-morina@nagasaki-u.ac.jp 
ability and no reference standard is available (Boeckh and Ljungman 2009; Halfon et al. 2011). A negative antigenemia result cannot rule out a CMV infection because this method shows low sensitivity compared to quantitative polymerase chain reaction (PCR) (Eguchi et al. 2017). Additionally, CMV antigenemia assay cannot be conducted in patients with leukopenia. Therefore, alternative suitable methods are needed to accurately evaluate the risk of CMV infection.

The World Health Organization (WHO) released the international reference standard for human CMV in 2010 (Freyer et al. 2010), based on which a quantitative PCR was developed (Ramanan and Razonable 2017). Targeting of CMV DNA in the pre-analysis process is advantageous because of the sample stability compared to during antigenemia (Nesbitt et al. 2004), and automated quantitative PCR can provide a broad linear range of results and short turnaround time (Tsai et al. 2016; Ramanan and Razonable 2017). Standardized nucleic acid tests (NATs) can reduce interlaboratory variability in CMV assays (Pang et al. 2009). A CMV NAT is preferred for diagnosis, decisions regarding preemptive therapy, and monitoring of the response to therapy in the management of hematopoietic stem cell transplantation (HSCT) (Boeckh and Ljungman 2009) and solid organ transplantation (Kotton et al. 2013; Razonable and Hayden 2013). However, no studies have examined the utility of standardized CMV NAT in Japanese transplant patients.

A semi-automated NAT test for detecting CMV DNA was developed to reduce technical variations and the risk of contamination. In Japan, antigenemia is a widely used standard method for CMV monitoring after transplantation. To determine the clinical utility of PCR-based CMV monitoring, the diagnosis performance of a semi-automated and standardized CMV NAT system was evaluated during antigenemia-guided CMV management after HSCT and liver transplantation.

\section{Materials and Methods}

\section{Study settings}

This study was conducted between October 1, 2013 and March 31, 2015 and was approved by the ethics committee of Nagasaki University Hospital. Written informed consent was obtained from each patient.

Patients who underwent HSCT or liver transplantation were included in this study. Age, sex, donor backgrounds (for HSCT, relatives/non-relatives and allograft/autograft; for liver transplantation, living donor/brain-dead donor), human leukocyte antigen (for HSCT) and $\mathrm{ABO}$ (for liver transplantation) compatibility, and CMV serological positivity were obtained as baseline characteristics. Data regarding prophylactic and preemptive therapy for CMV infections and the findings for graft-versus-host disease (GVHD) were also obtained.

\section{Sample collection and CMV testing}

Blood collection was performed under the physician's instructions depending on the condition of the patient. Patients were monitored for CMV pp65 antigenemia (from weekly to monthly), and the blood samples for CMV NAT were simultaneously obtained and stored at $-80^{\circ} \mathrm{C}$ until evaluation. For HSCT, only CMV NAT was performed during the pre-engraftment period. Monitoring was continued until 6 months after transplantation or until discontinuation of follow-up in our hospital.

For CMV pp65 antigenemia, blood specimens were collected in a sampling tube containing EDTA-2Na. CMV antigenemia was tested by an external clinical laboratory company, and the results were used for typical CMV management. The results were plotted as the number of positive cells among 50,000 cells.

For CMV NAT, blood ( $2 \mathrm{~mL}$ ) was collected into another sampling tube containing EDTA-2K and plasma was stored at $-80^{\circ} \mathrm{C}$ until analysis. DNA was extracted from $500 \mu \mathrm{L}$ of plasma using COBAS AmpliPrep (Roche Diagnostics, Basel, Switzerland), and the CMV UL54 gene which encodes DNA polymerase was amplified with the COBAS TaqMan CMV Test (Roche Diagnostics) according to the manufacturer's protocol. This system is automated for the amplification and detection of CMV UL54. The internal control was also automatically evaluated in all samples and negative and positive controls were measured in each assay. The quantification range was 150 to $1 \times 10^{7}$ copies $/ \mathrm{mL}$. When UL54 was amplified but the copy number was less than 150 copies $/ \mathrm{mL}$, the result was expressed as $<1.50 \times 10^{2}$ copies $/ \mathrm{mL}$. The physicians were blinded to the results of the NAT.

\section{Results}

\section{CMV monitoring in HSCT recipients}

A total of 11 HSCT recipients were enrolled in the study (Table 1). The median age was 55 years (range, $18-66)$, and 3 patients $(27.3 \%)$ were men. All patients were allograft-transplanted, $6(45.5 \%)$ were transplanted from relatives, and $5(45.5 \%)$ were HLA-compatible at both the serum and DNA levels. Regarding the serological conditions (recipient ${ }^{+/}$donor $^{+/}$) before transplantation, the numbers of $\mathrm{R}^{+} \mathrm{D}^{+}, \mathrm{D}^{+} \mathrm{R}^{-}, \mathrm{D}^{-} \mathrm{R}^{+}$, and $\mathrm{D}^{-} \mathrm{R}^{-}$were $3,4,1$, and 0 , respectively. All patients were prescribed with anti-CMV drugs.

The time series of the results were compared (Fig. 1). Six patients were positive for CMV in both tests during the study period (Patients 1-6). Three patients were positive for CMV only in NAT (Patients 7-9), and the remaining 2 patients were negative (Patients 10 and 11). In 4 patients, CMV NAT was positive during the pre-engraftment period (Patients 2, 4, 8, and 9).

In 2 patients, NAT and antigenemia simultaneously detected the first CMV-positive episode (Patients 1 and 2). NAT detected CMV earlier than antigenemia in 3 patients (Patients 3-5) but 3 weeks later than antigenemia in 1 patient (Patient 6).

Four patients were intermittently positive in antigenemia (Patients 1, 3, 4, and 6); however, NAT detected CMV between the antigenemia-positive episodes.

Eight patients were administered anti-CMV treatment (Fig. 2), all of whom had a history of GVHD. Of these patients, 7 were positive for CMV in either test (Patients 1-6, and 8); however, the values were near the detection limit except for in 2 patients (Patients 1 and 2). In 1 patient, 
Table 1. Baseline characteristics of HSCT recipients.

\begin{tabular}{lc}
\hline \multicolumn{1}{c}{ Characteristics } & HSCT recipients \\
\hline Median age, years old (range) & $55(18-66)$ \\
Sex, male, $\mathrm{n}(\%)$ & $3(27.3)$ \\
Source for transplant, $\mathrm{n}(\%)$ & \\
$\quad$ Bone marrow & $2(18.2)$ \\
Peripheral blood & $6(54.6)$ \\
Umbilical cord blood & $3(27.3)$ \\
Allograft transplant, $\mathrm{n}(\%)$ & $11(100.0)$ \\
Transplant from relatives, $\mathrm{n}(\%)$ & $5(45.5)$ \\
Serostatus, $\mathrm{n}(\%)$ & \\
$\mathrm{R}^{+} \mathrm{D}^{+}$ & $3(27.3)$ \\
$\mathrm{R}^{+} \mathrm{D}^{-}$ & $4(36.4)$ \\
$\mathrm{R}^{-} \mathrm{D}^{+}$ & $1(9.1)$ \\
$\mathrm{R}^{-} \mathrm{D}^{-}$ & $0(0.0)$ \\
Unknown & $3(27.3)$ \\
HLA compatibility, $\mathrm{n}(\%)$ & \\
Compatible in serum and DNA levels & $5(45.5)$ \\
\hline
\end{tabular}

$\mathrm{R}$, recipient; $\mathrm{D}$, donor.

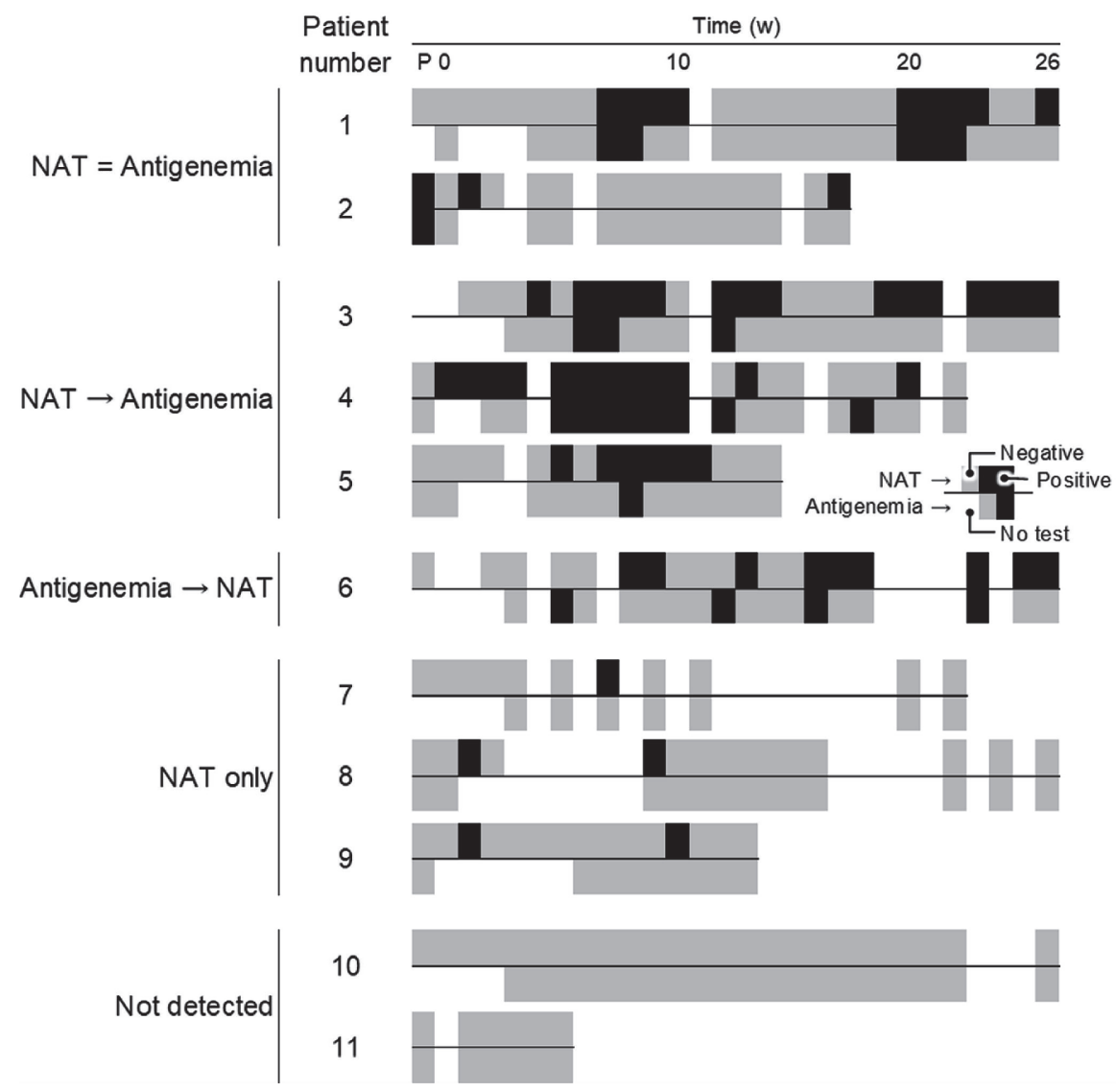

Fig. 1. CMV monitoring of HSCT recipients over time.

The weekly results of NAT (upper lane) and antigenemia (lower lane) are presented for each HSCT recipient. Each box indicates a representative weekly result of the CMV test (black, positive; gray, negative; blank, no test). The NAT detected CMV no later (Patients 1 and 2), earlier (Patients 3-5), and later (Patient 6) than antigenemia. CMV was also detected by NAT only (Patients 7-9) and not detected by both tests (Patients 10 and 11).

$\mathrm{P}$, pre-transplantation. 

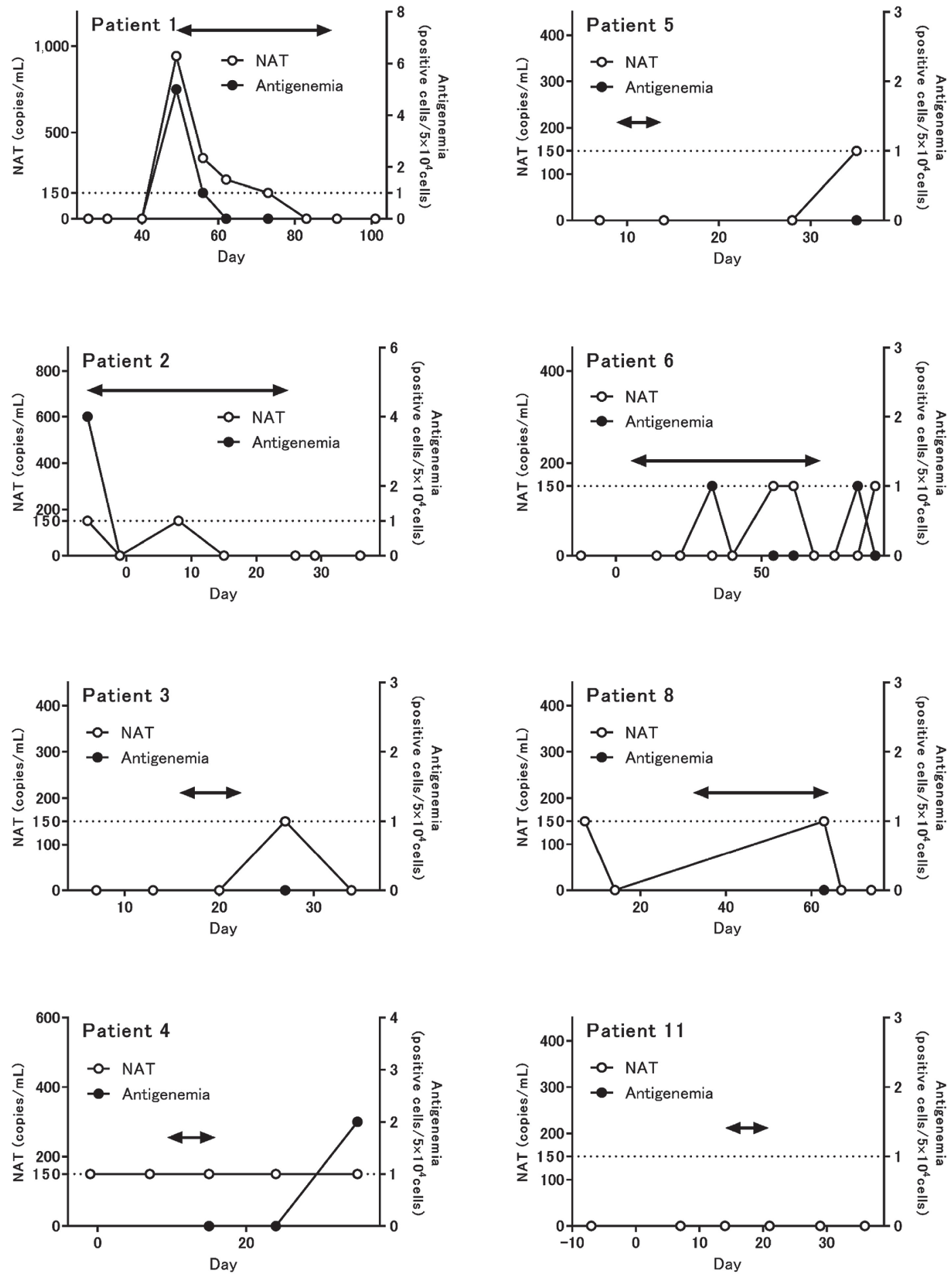

Fig. 2. Time courses of CMV monitoring and anti-CMV treatment in HSCT recipients.

The time series of antigenemia (filled circle) and NAT (open circle) are displayed for cases with anti-CMV treatment. Double arrow represents the period of anti-CMV preemptive therapy.

NAT still showed a positive result after the antigenemia results became negative (Patient 1 in Fig. 2).

\section{CMV monitoring in liver transplant recipients}

A total of 23 liver transplant recipients were enrolled (Table 2). The median age was 59 years (range, 12-70), and 12 patients $(52.3 \%)$ were men. Of these patients, 22
(95.7\%) were transplanted from living donors and 1 (4.3\%) was from a brain-dead donor. Seventeen (73.9\%) patients were ABO-compatible, and all donor-recipient pairs were CMV-seropositive. Twenty-one (91.3\%) patients were prescribed with anti-CMV drugs.

The time series of monitoring was compared (Fig. 3). A total of 16 (69.6\%) patients showed CMV-positive results 
Table 2. Baseline characteristics of liver transplant recipients.

\begin{tabular}{lc}
\hline \multicolumn{1}{c}{ Characteristics } & Liver transplant recipients \\
\hline Median age, years old (range) & $59(12-70)$ \\
Sex, male, $\mathrm{n}(\%)$ & $12(52.3)$ \\
Donor, $\mathrm{n}(\%)$ & \\
$\quad$ Living & $22(95.7)$ \\
$\quad$ Brain dead & $1(4.3)$ \\
Recipient serostatus & \\
$\quad$ Positive & $23(100.0)$ \\
ABO compatibility & \\
$\quad$ Compatible & $17(73.9)$ \\
\hline
\end{tabular}

in NAT and/or antigenemia during the study period (Patients 12-27). Four (17.4\%) patients were positive for CMV only in NAT (Patients 23-26) and 1 (4.3\%) was positive for CMV only in antigenemia (Patient 27). The remaining 7 patients $(30.4 \%)$ had no sign of CMV in both tests (Patients 28-32).

In 3 of the 12 patients who showed positive results in both tests, NAT and antigenemia simultaneously detected the first CMV-positive episode (Patients 12-14). NAT detected CMV earlier than antigenemia in 7 patients (Patients 15-21) but 1 week later than antigenemia in 1 patient (Patient 22).

Six patients were intermittently positive in antigenemia; however, NAT detected CMV between the antigenemia-positive episodes (Patients 13, 15, 16, 18, 20, and 21).

Anti-CMV drugs were administered to 7 patients as preemptive therapy (Fig. 4). The CMV levels in NAT and antigenemia decreased after treatment was started. In 3 patients, antigenemia showed negative results earlier than in the NAT (Patients 13, 17, and 18).

\section{Test performance of CMV NAT}

The qualitative correlation between CMV NAT and antigenemia was evaluated. In the samples collected from the 34 patients, 468 samples showed both results. Of these samples, NAT and antigenemia test detected CMV in 124 $(26.7 \%)$ and $51(10.9 \%)$ samples, respectively (Table 3$)$. The concordance between the two tests was $80.1 \%$.

Next, quantitative correlation between the two tests was evaluated using the same 468 samples. The number of NAT-positive samples ranged from $<1.50 \times 10^{2}$ to $2.31 \times$ $10^{3}$ copies $/ \mathrm{mL}$ and those of antigenemia ranged from 1 to 12 cells/ $5 \times 10^{4}$ cells. The coefficient of determination between the two tests was 0.303 (Fig. 5).

There were 93 discordant samples (Table 3). In 73 $(88.0 \%)$ of the 83 NAT-positive and antigenemia-negative samples, NAT revealed $<1.50 \times 10^{2}$ copies $/ \mathrm{mL}$ and the viral load of the remaining 10 samples ranged from $1.92 \times$ $10^{2}$ to $1.20 \times 10^{3} \mathrm{copies} / \mathrm{mL}$. In the 10 NAT-negative samples, antigenemia detected 1 or 2 positive cells $/ 5 \times 10^{4}$ cells.

\section{Discussion}

Accurately detecting CMV is important for transplant recipients because these patients have several risk factors such as GVHD and immunosuppressive therapy (ArizaHeredia et al. 2014; Marcelin et al. 2014). At the analytical level, the stability of CMV DNA can contribute to reducing inconsistencies during pre-analysis and produce reliable results (Nesbitt et al. 2004). Additionally, CMV NAT can be standardized for improving the management of CMV. Similar to previous reports (Halfon et al. 2011; Ishii et al. 2017), the NAT and antigenemia showed nearly the same results for CMV infection; however, the NAT also showed the early detection and delayed clearance of CMV compared to antigenemia. Apparent disadvantages due to discordances between NAT and antigenemia were not observed in the present study, suggesting that NAT can be used as an alternative method for CMV monitoring.

NAT detected CMV more frequently than antigenemia, and most events that only showed a positive result in the antigenemia test were accompanied by positive NAT results at the nearest time point. These results suggest that the efficacy of NAT for detecting CMV is equivalent to or superior to that of antigenemia. In contrast, this finding also suggests that CMV can be over-diagnosed by NAT compared to analysis by antigenemia. However, early detection by CMV NAT can indicate two possibilities for CMV management. First, CMV NAT can be used to determine preemptive treatment earlier than antigenemia-based CMV management. Second, CMV NAT can provide an appropriate cut-off value for starting empiric CMV treatment because anti-CMV therapy was not always necessary when the NAT indicated the lowest viral load $\left(<1.50 \times 10^{2}\right.$ copies $\left./ \mathrm{mL}\right)$. To determine whether these strategies lead to successful CMV management compared to antigenemia-guided management, further clinical studies are required.

For HSCT recipients, prophylaxis can reduce CMV infections during the post-engraftment phase (Sahin et al. 2016). However, whether CMV positivity during the preengraftment phase indicates a risk of CMV infections remains unclear (Ullmann et al. 2016). The present study supported that CMV DNAemia occurs during the pre- 

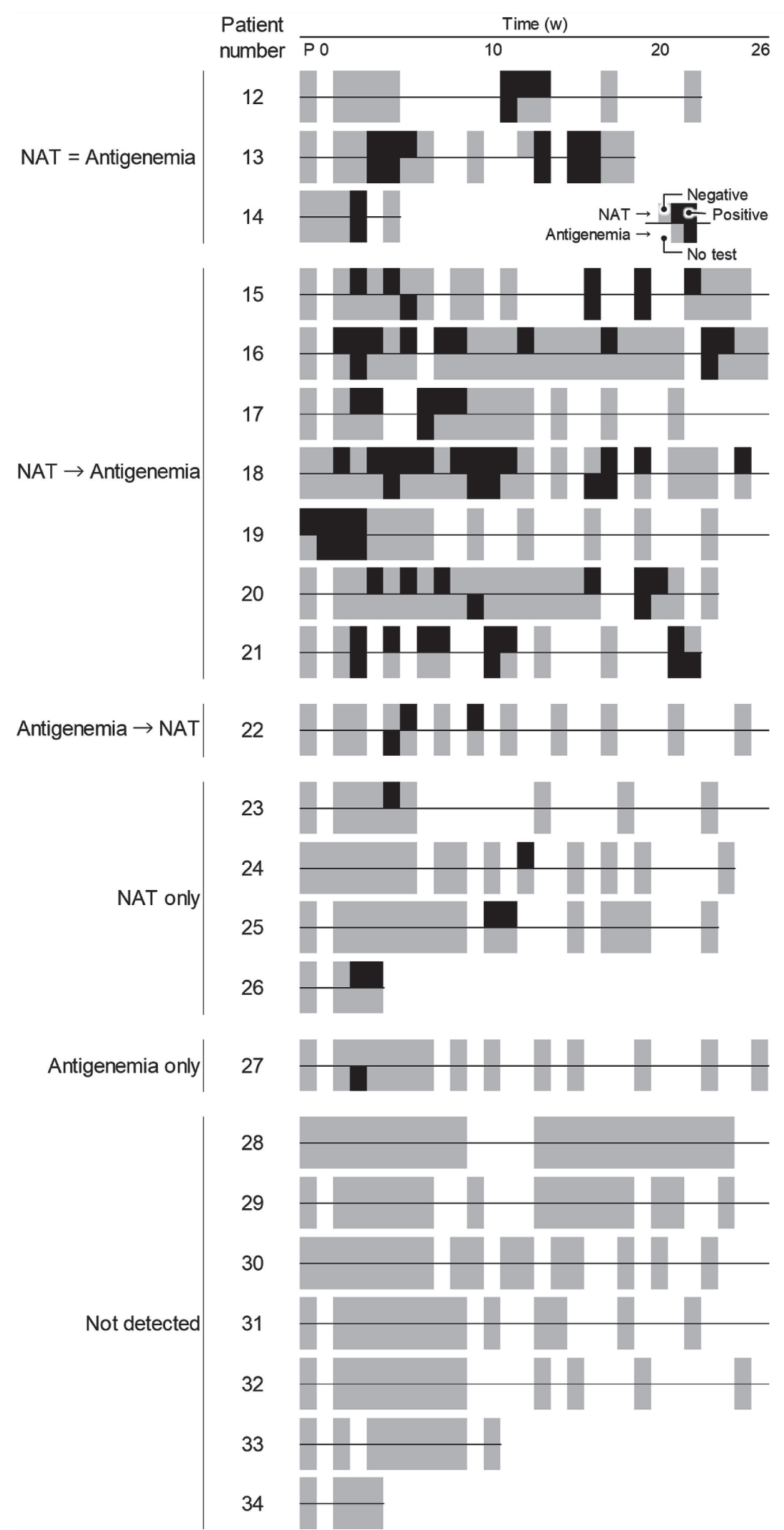

Fig. 3. Time series of CMV monitoring of liver transplant recipients.

The weekly results of NAT (upper lane) and antigenemia (lower lane) are presented for each liver transplant recipient. Each box represents the weekly result, as presented in Fig. 1. The NAT detected CMV no later (Patients 12-14), earlier (Patients 15-21), and later (Patient 22) than antigenemia. Patients with NAT-positive only (Patients 23-26) and antigenemia-positive only (Patient 27) results and those with no detection by both tests (Patients 28 and 34) were also observed. $\mathrm{P}$, pre-transplantation. 

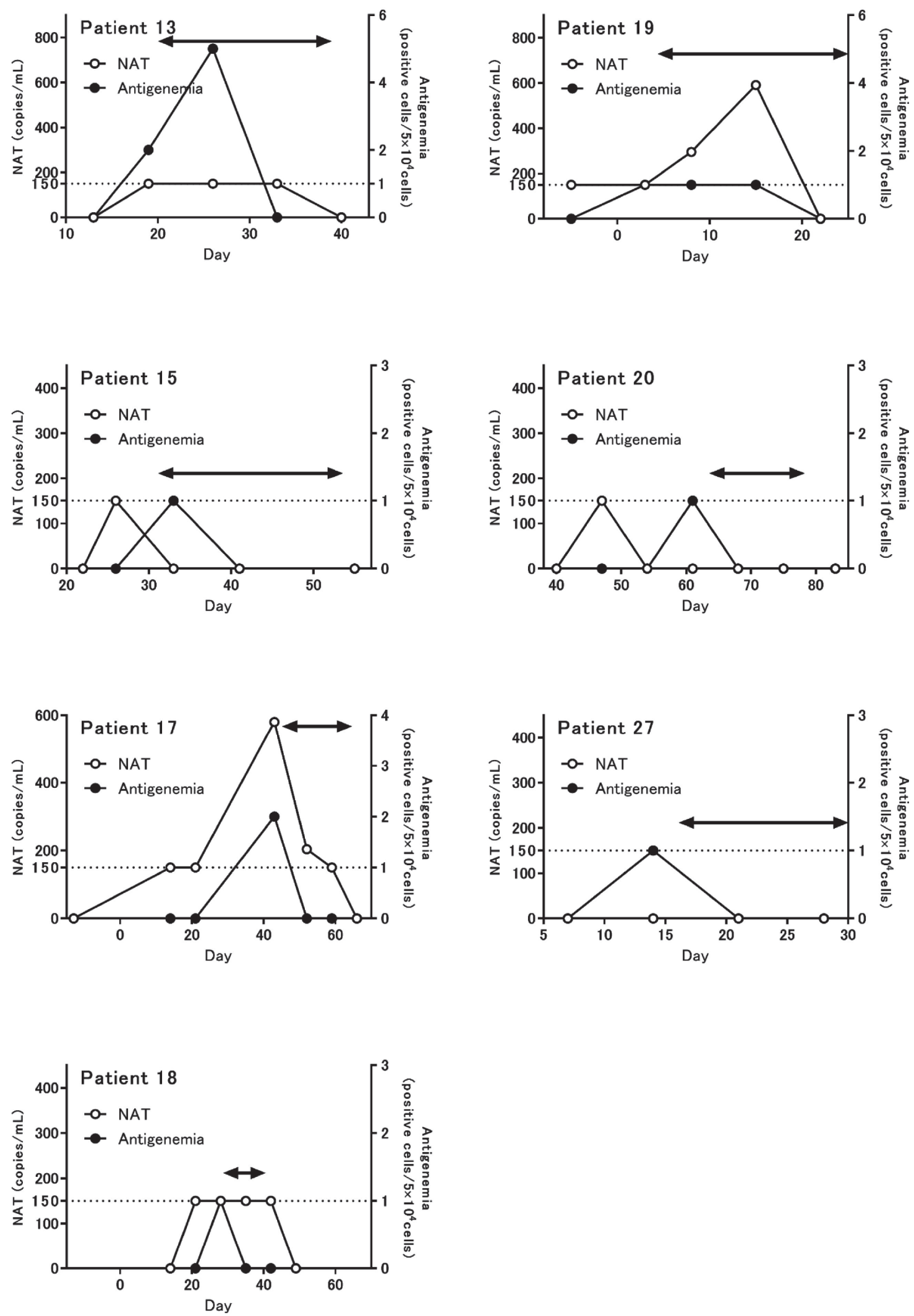

Fig. 4. Time series of CMV monitoring and anti-CMV treatment of liver transplant recipients.

The time series of antigenemia (filled circle) and the NAT (open circle) are displayed for cases with anti-CMV treatment. Double arrow represents the period of anti-CMV preemptive therapy. 
Table 3. Testing performances of NAT compared with antigenemia.

\begin{tabular}{ccccc}
\hline & & \multicolumn{2}{c}{ Antigenemia } & Total \\
\cline { 3 - 4 } & & Positive & Negative & \\
\hline \multirow{3}{*}{ NAT } & Positive & 41 & 83 & 124 \\
& Negative & 10 & 34 & 344 \\
\hline & & 51 & 417 & 468 \\
\hline
\end{tabular}

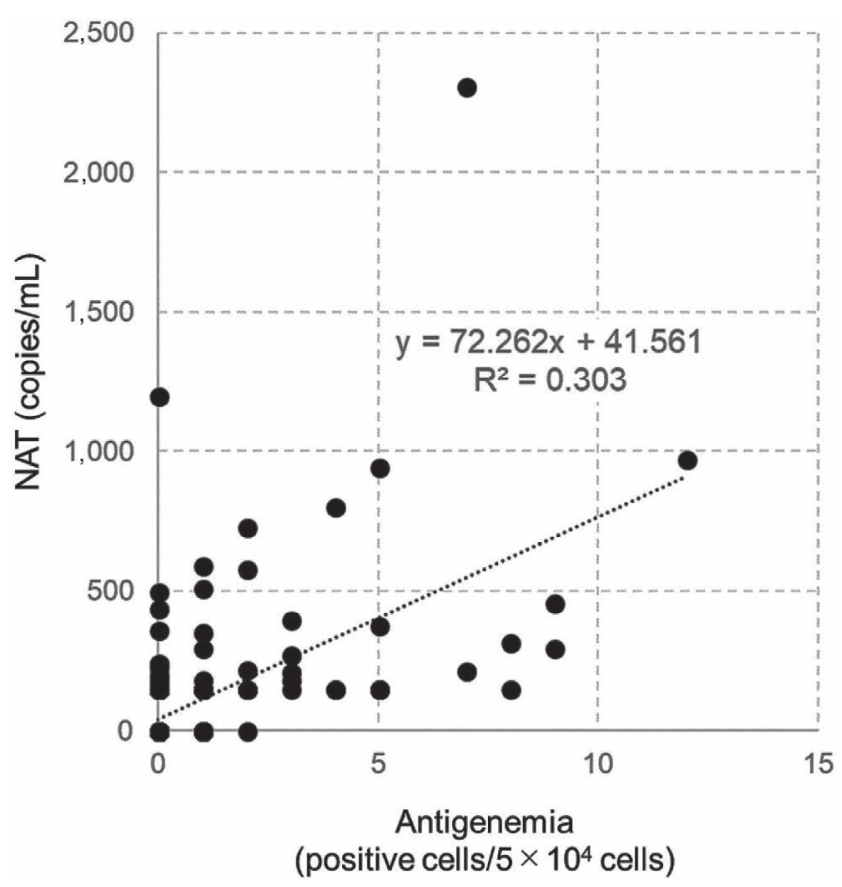

Fig. 5. Correlation between NAT and antigenemia.

The quantitative correlation between the results of the NAT and antigenemia test in 468 blood samples which were evaluated by both tests. Each dot indicates one sample, and several dots overlap because of the same results. The results of NAT $<1.50 \times 10^{2}$ copies $/ \mathrm{mL}$ were plotted as $1.50 \times 10^{2}$ copies $/ \mathrm{mL}$.

engraftment phase. Generally, CMV infections are rarely observed during this phase (Kedia et al. 2013; Sahin et al. 2016; Fabiani et al. 2017); however, the continuous NATpositive results during the pre-engraftment phase indicate a considerable risk of CMV infection after engraftment (patient 4).

The equipment used in the present study is semi-automated and can assess multiple samples simultaneously. CMV NAT requires costly equipment for use in clinical settings. Therefore, CMV NAT may be suitable for use in specific medical facilities where transplantation therapies are aggressively performed. Alternatively, some core medical laboratories can perform NAT upon receiving specimens from several medical institutions because CMV DNA is relatively stable.

There were some limitations to this study. First, because this study evaluated a small number of patients, further analysis is required to identify the characteristics of NAT. Second, this study did not include reference methods for evaluating the performance of NAT because another standardized method was unavailable during the study period. Finally, to determine the advantage of standardization, a multi-center study should be conducted.

Standardization of CMV testing will support wellguided treatment strategies and provide a reliable comparison between facilities. Standardized CMV NAT can also enhance the quality of CMV testing algorithms such as therapeutic cutoffs and testing frequencies. Thus, the CMV NAT can improve CMV management for transplant recipients.

\section{Acknowledgments}

This study was supported by Health and Labor Sciences Research Grants from the Ministry of Health, Labour, and Welfare in Japan (H28-Shinkou-Ippan-003); a grant for research and development of diagnostic methods and therapies for antimicrobial-resistant bacteria from Japan Agency for Medical Research and Development (JP18fk0108052). This study was also funded by Roche Diagnostics K.K. (Tokyo, Japan).

\section{Author Contributions}

Y. Morinaga participated in research design, performance of the research, data analysis, and writing of the article. Y.S., M.H., J.T., and M.T. participated in the research design and performance of the research. S.M participated in the performance of the research and data analysis. S.E., Y. Miyazaki, and K.Y. participated in the research design and writing of the article.

\section{Conflict of Interest}

The authors declare no conflict of interest.

\section{References}

Ariza-Heredia, E.J., Nesher, L. \& Chemaly, R.F. (2014) Cytomegalovirus diseases after hematopoietic stem cell transplantation: a mini-review. Cancer Lett., 342, 1-8.

Boeckh, M. \& Ljungman, P. (2009) How we treat cytomegalovirus in hematopoietic cell transplant recipients. Blood, 113, 57115719.

Eguchi, H., Horita, N., Ushio, R., Kato, I., Nakajima, Y., Ota, E. \& Kaneko, T. (2017) Diagnostic test accuracy of antigenaemia assay for PCR-proven cytomegalovirus infection-systematic review and meta-analysis. Clin. Microbiol. Infect., 23, 907-915.

Fabiani, S., Fortunato, S., Petrini, M. \& Bruschi, F. (2017) Allogeneic hematopoietic stem cell transplant recipients and parasitic diseases: a review of the literature of clinical cases and perspectives to screen and follow-up active and latent chronic infections. Transpl. Infect. Dis., 19.

Freyer, J., Heath, A., Anderson, R. \& Minor, P. (2010) Collaborative study to evaluate the proposed 1 st WHO international standard for human cytomegalovirus for nucleic acid amplification-based assays. WHO ECBS Report, 1-40.

Halfon, P., Berger, P., Khiri, H., Martineau, A., Pénaranda, G., 
Merlin, M. \& Faucher, C. (2011) Algorithm based on CMV kinetics DNA viral load for preemptive therapy initiation after hematopoietic cell transplantation. J. Med. Virol., 83, 490-495.

Ishii, K., Onishi, Y., Miyamura, N., Fukuhara, N., Ishizawa, K., Nakanishi, M., Ohnaka, S., Miyasaka, T., Kanno, E., Kawakami, K., Harigae, H. \& Kaku, M. (2017) Development and evaluation of a quantitative assay detecting cytomegalovirus transcripts for preemptive therapy in allogeneic hematopoietic stem cell transplant recipients. J. Med. Virol., 89, $1265-1273$.

Kedia, S., Acharya, P., Mohammad, F., Nguyen, H., Asti, D., Mehta, S., Pant, M. \& Mobarakai, N. (2013) Infectious complications of hematopoietic stem cell transplantation. $J$. Stem Cell Res. Ther., S3, 002.

Kotton, C.N., Kumar, D., Caliendo, A.M., Asberg, A., Chou, S., Danziger-Isakov, L. \& Humar, A.; Transplantation Society International, CMV Consensus Group (2013) Updated international consensus guidelines on the management of cytomegalovirus in solid-organ transplantation. Transplantation, 96, 333-360.

Marcelin, J.R., Beam, E. \& Razonable, R.R. (2014) Cytomegalovirus infection in liver transplant recipients: updates on clinical management. World J. Gastroenterol., 20, 10658-10667.

Nesbitt, S.E., Cook, L. \& Jerome, K.R. (2004) Cytomegalovirus quantitation by real-time PCR is unaffected by delayed separation of plasma from whole blood. J. Clin. Microbiol., 42,
1296-1297.

Pang, X., Fox, J., Fenton, J., Miller, G., Caliendo, A. \& Preiksaitis, J.K.; American Society of Transplantation Infectious Diseases Community of Practice; Canadian Society of Transplantation (2009) Interlaboratory comparison of cytomegalovirus viral load assays. Am. J. Transplant., 9, 258-268.

Ramanan, P. \& Razonable, R.R. (2017) Evaluation of COBAS AmpliPrep/COBAS TaqMan CMV Test for use in hematopoietic stem cell transplant recipients. Expert Rev. Mol. Diagn., 17, 633-639.

Razonable, R.R. \& Hayden, R.T. (2013) Clinical utility of viral load in management of cytomegalovirus infection after solid organ transplantation. Clin. Microbiol. Rev., 26, 703-727.

Sahin, U., Toprak, S.K., Atilla, P.A., Atilla, E. \& Demirer, T. (2016) An overview of infectious complications after allogeneic hematopoietic stem cell transplantation. J. Infect. Chemother, 22, 505-514.

Tsai, H.P., Tsai, Y.Y., Lin, I.-T., Kuo, P.H., Chen, T.Y., Chang, K.C. \& Wang, J.R. (2016) Comparison of two commercial automated nucleic acid extraction and integrated quantitation realtime PCR platforms for the detection of cytomegalovirus in plasma. PLoS One, 11, e0160493.

Ullmann, A.J., Schmidt-Hieber, M., Bertz, H., Heinz, W.J., Kiehl, M., Krüger, W., Mousset, S., Neuburger, S., Neumann, S. \& Penack, O. (2016) Infectious diseases in allogeneic haematopoietic stem cell transplantation: prevention and prophylaxis strategy guidelines 2016. Ann. Hematol., 95, 1435-1455. 